\title{
Association between Body Weight Gain from 20 Years of Age and Diabetic Nephropathy in Japanese Patients with Type 2 Diabetes
}

\author{
Hiroshi Okada ${ }^{a}$ Muhei Tanakab Goji Hasegawac Hanako Nakajimad \\ Mayuko Kadono $^{c}$ Tomoki Miyoshia $^{a}$ Yuki Okada $^{a}$ Koji Nakano ${ }^{\mathrm{e}}$ \\ Tetsuro Yamane $^{\text {f }}$ Michiaki Fukuid $^{d}$ \\ aDepartment of Diabetes and Endocrinology, Matsushita Memorial Hospital, Moriguchi, \\ Japan; ${ }^{b}$ Department of Internal Medicine, Kyotamba Hospital, Kyotamba, Japan; \\ 'Department of Diabetes, Endocrinology, Nephrology and Rheumatology, Japanese Red \\ Cross Kyoto Daini Hospital, Kyoto, Japan; ${ }^{\mathrm{d} D e p a r t m e n t ~ o f ~ E n d o c r i n o l o g y ~ a n d ~ M e t a b o l i s m, ~}$ \\ Kyoto Prefectural University of Medicine, Graduate School of Medical Science, Kyoto, Japan; \\ e Department of Endocrinology and Metabolism, Kyoto Yamashiro General Medical Center, \\ Kyoto, Japan; fDepartment of Surgery, Matsushita Memorial Hospital, Moriguchi, Japan
}

\section{Keywords}

Body weight gain $\cdot$ Nephropathy $\cdot$ Type 2 diabetes

\begin{abstract}
Background/Aims: It has been reported that the body mass index shows a U-shaped association with death from cardiovascular disease (CVD) in the Asian population. The relationship between body weight (BW) gain from early adulthood and diabetic nephropathy remains to be elucidated in Japanese patients with type 2 diabetes. Our aim was to investigate the association between BW gain from early adulthood and diabetic nephropathy in Japanese patients with type 2 diabetes. Methods: We assessed the BW of 471 consecutive patients with type 2 diabetes and calculated the change in BW from the age of 20 years to the lifetime maximum $\left(\triangle B W_{\max -20 y}\right)$. We then evaluated the relationship of $\triangle B W_{\text {max-20y }}$ with the degree of urinary albumin excretion (UAE), which is a useful marker for CVD. Results: $\triangle B W_{\text {max-20y }}$ negatively correlated with the logarithm of UAE $(r=-0.160, p=0.002)$. Multiple regression analysis demonstrated that $\triangle B W_{\max -20 y}$ was independently correlated with the logarithm of UAE $(\beta=-0.112, p=0.034)$. Conclusions: BW gain from the age of 20 years is correlated with diabetic nephropathy in Japanese patients with type 2 diabetes.




\section{Kidney \\ Blood Pressure \\ Research}

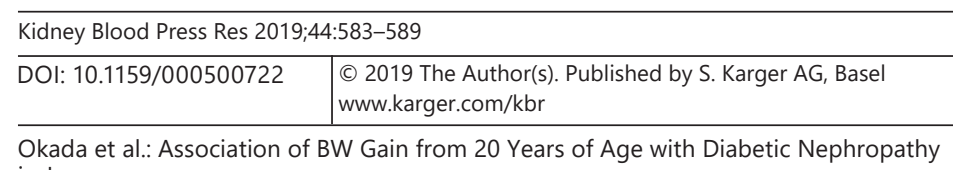

in Japan

\section{Introduction}

Cardiovascular disease (CVD) is the primary cause of mortality and morbidity in patients with type 2 diabetes, and several risk factors including smoking, hypertension, and dyslipidemia have been shown to accelerate the progression of CVD [1,2]. Elevated albumin excretion rate, which is a useful marker for diabetic nephropathy, has also been reported to be associated with an increased risk of cardiovascular mortality and the progression of CVD $[3,4]$.

The prevalence of overweight and obesity, which have been increasing worldwide, are well-established risk factors for diabetes, hypertension, and CVD [5-10]. On the other hand, it has been reported that body mass index (BMI) shows a U-shaped association with death from CVD in the Asian population [11,12]. Across BMI categories, the hazard ratio of CVD did not change significantly in the Asian population with diabetes [13]. Higher BMI might be a weak risk factor for mortality from CVD in the Asian population, for which a lower prevalence of overweight or obesity compared with Western populations has been reported [11]. A few studies have demonstrated the association between body weight (BW) status and CVD in diabetic patients. Carnethon et al. [14] found an association of BW status with CVD in adults with incident diabetes; they reported that adults of normal weight at the time of incident diabetes had higher cardiovascular mortality than those who were overweight or obese. Bello et al. [15] demonstrated that BW gain in the short term could exacerbate albuminuria. However, the relationship between BW gain from early adulthood and diabetic complications, including albuminuria, remains to be elucidated in Asian patients with type 2 diabetes. We evaluated the relationship between BW gain from early adulthood and albuminuria in Japanese patients with type 2 diabetes.

\section{Methods}

\section{Patients and Study Design}

We performed a cross-sectional study on 471 patients with type 2 diabetes recruited from the outpatient clinic at the Matsushita Memorial Hospital, Japanese Red Cross Kyoto Daini Hospital, and Kyoto Yamashiro General Medical Center from April 2014 to July 2017. Type 2 diabetes was diagnosed according to the Report of the Expert Committee on the Diagnosis and Classification of Diabetes Mellitus [16]. BMI was calculated. Patients were classified as nonsmokers, past smokers, or current smokers based on information in a self-administered questionnaire. Patients with advanced renal dysfunction (serum creatinine [Cr] $>2.0 \mathrm{mg} / \mathrm{dL}$ ), malignancy, or a psychological disorder were excluded from the study. We then evaluated the relationship of BW gain from early adulthood to albuminuria in all patients.

\section{Patients and Public Involvement}

Our study participants were members of the general population with type 2 diabetes. The research questions and outcome measures were developed to guide future clinical practice and public management. No patients were involved in the design of, recruitment to, or conducting of the study. Patients at the Matsushita Memorial Hospital can assess results of the hospital's studies via the website.

\section{Assessment of BW Gain from Early Adulthood and Data Collection}

BW at 20 years and maximum lifetime BW were obtained from a database containing information from self-administered questionnaires. BW gain from early adulthood was defined as BW change from 20 years to maximum lifetime $B W\left(\Delta B W_{\max -20 y}\right)$. 


\section{Kidney \\ Blood Pressure \\ Research}

Table 1. Characteristics of patients

\begin{tabular}{|c|c|}
\hline \multicolumn{2}{|c|}{ Kidney Blood Press Res 2019;44:583-589 } \\
\hline DOI: $10.1159 / 000500722$ & $\begin{array}{l}\text { (c) } 2019 \text { The Author(s). Published by S. Karger AG, Basel } \\
\text { www.karger.com/kbr }\end{array}$ \\
\hline
\end{tabular}

$471(295 / 176)$

$66.4(10.7)$

$13.0(9.6)$

$24.3(3.8)$

$22.5(3.7)$

$27.7(4.2)$

$131.8(18.6)$

$7.5(1.3)$

$4.67(0.82)$

$1.69(1.04)$

$321.2(77.3)$

$72.7(22.0)$

$230 / 149 / 92$

$14.6(6.5-35.4)$

$253 / 219$

$152 / 43 / 207$

$207 / 264$

Data are expressed as $n$, mean (SD), or median (interquartile range). RAS, renin-angiotensin system.

Overnight fasting blood samples were taken in the morning. Serum total cholesterol and triglyceride concentrations were assessed using standard enzymatic methods. Hemoglobin $\mathrm{A}_{1 \mathrm{c}}\left(\mathrm{HbA}_{1 \mathrm{c}}\right)$ was assayed using high-performance liquid chromatography and expressed as the unit defined by the National Glycohemoglobin Standardization Program. Urinary albumin and $\mathrm{Cr}$ concentrations were determined in early morning spot urine. Urinary albumin excretion (UAE) was measured using an immunoturbidimetric assay.

\section{Statistical Analysis}

Means, medians, and frequencies of potential confounding variables were calculated. Because triglycerides and UAE showed skewed distributions, log transformation was carried out before performing correlation and regression analysis. The relationships between $\triangle \mathrm{BW}_{\text {max-20y }}$ and the logarithm of UAE, and between $\Delta \mathrm{BW}_{\max -20 \mathrm{y}}$ and age or other variables were examined by Pearson's correlation analyses. To examine the effects of various factors on the logarithm of UAE, the following were considered simultaneously as independent variables for multiple regression analysis: age, sex, BMI, duration of diabetes, $\Delta B W_{\text {max }} 20 y$, average systolic blood pressure (SBP), $\mathrm{HbA}_{1 \mathrm{c}}$, total cholesterol, the logarithm of triglyceride, uric acid, $\mathrm{Cr}$, smoking status, the use of dipeptidyl peptidase- 4 inhibitors, renin-angiotensin system (RAS) inhibitors, and statin. All continuous variables are presented as mean (standard deviation), median (interquartile range), or absolute number. A $p$ value $<0.05$ was considered statistically significant.

\section{Results}

Characteristics of the 471 patients with diabetes enrolled in this study are shown in Table 1. Mean current BMI, BMI at 20 years, or maximum lifetime BMI were 24.3 (3.8), 22.5 (3.7), and 27.7 (4.2), respectively. The median UAE was 14.6 (6.5-35.4) $\mathrm{mg} / \mathrm{g} \mathrm{Cr}$. 


\section{Kidney \\ Blood Pressure \\ Research}

Table 2. Correlation between body weight gain from the age of 20 years and other variables

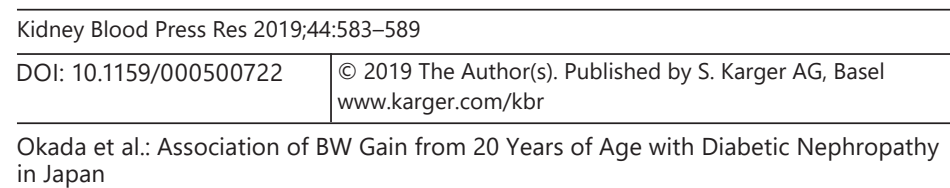

\begin{tabular}{lrr}
\hline & \multicolumn{2}{c}{ Body weight gain; } \\
& \multicolumn{2}{c}{$\Delta \mathrm{BW}_{\text {max }-20 \mathrm{y}}$} \\
\cline { 2 - 3 } & \multicolumn{2}{c}{$p$} \\
\hline Age & -0.172 & 0.0002 \\
Body mass index & 0.445 & $<0.0001$ \\
Duration of diabetes & -0.131 & 0.004 \\
Average systolic blood pressure & -0.035 & 0.448 \\
Hemoglobin $\mathrm{A}_{1 \mathrm{c}}$ & -0.084 & 0.069 \\
Total cholesterol & 0.011 & 0.808 \\
Logarithm of triglyceride & 0.086 & 0.063 \\
Uric acid & 0.021 & 0.647 \\
Creatinine & -0.064 & 0.134 \\
Logarithm of urinary albumin excretion & -0.160 & 0.002 \\
\hline
\end{tabular}

No significant correlations were found between current BMI, BMI at 20 years, or maximum lifetime BMI and the logarithm of UAE. Relationships between $\triangle B W_{\max -20 \mathrm{y}}$ and other variables are shown in Table 2. No significant correlations were found between $\Delta \mathrm{BW}_{\max -20 \mathrm{y}}$ and average $\mathrm{SBP}, \mathrm{HbA}_{1 \mathrm{c}}$, total cholesterol, the logarithm of triglyceride, uric acid, or $\mathrm{Cr} . \Delta \mathrm{BW}_{\text {max-20y }}$ was positively correlated with BMI $(r=0.445, p<0.0001) . \Delta \mathrm{BW}_{\text {max-20y }}$ was negatively correlated with age $(r=-0.172, p=0.0002)$, duration of diabetes $(r=-0.131, p=0.004)$, and the logarithm of UAE $(r=-0.160, p=0.002)$.

Simple correlation and multiple regression analysis on the logarithm of UAE are shown in Table 3. Multiple regression analysis demonstrated that $\Delta \mathrm{BW}_{\max -20 \mathrm{y}}$ was independently correlated with the logarithm of UAE $(\beta=-0.112, p=0.034)$.

\section{Discussion}

The major finding of our study was that there were significant correlations between BW gain from early adulthood and albuminuria in Japanese patients with type 2 diabetes. BW gain from early adulthood was negatively correlated with the logarithm of UAE. The negative correlation between BW gain from early adulthood and albuminuria was unexpected. Our findings could reflect a higher UAE in patients who already had risk factors unrelated to diabetes. However, the findings persisted after adjustment for known risk factors such as duration of diabetes, hypertension, or smoking.

Although unintentional BW loss is common in patients with type 1 diabetes, it is also present in type 2 diabetes [17]. Guidelines recommend intentional BW loss for better glycemic control and an improvement in other CVD risk factors in type 2 diabetes patients [18]. Both scenarios are potentially controversial for describing the association between BW gain from early adulthood and albuminuria. Apparent $\beta$ cell destruction might cause unintentional BW loss. In this study, we did not include patients with type 1 diabetes who had $\beta$ cell destruction. However, we did not identify the exact type of diabetes in all patients owing to the difficulty of accessing the data on C-peptide or glutamic acid decarboxylase. Therefore, we could not determine whether patients with little change in BW had $\beta$ cell destruction, e.g., latent autoimmune diabetes in adults [19]. Little change in BW from early adulthood might be caused by impaired insulin secretion. The duration of impaired glucose tolerance in patients who have little change in BW from early adulthood might be longer than patients who did not have little change in BW. 
Table 3. Correlation between logarithm of urinary albumin excretion and other variables

\begin{tabular}{lcccc}
\hline & $r$ & $p$ & $\beta$ & $p$ \\
\hline Age & 0.178 & 0.0006 & 0.041 & 0.418 \\
Sex & - & - & -0.189 & 0.001 \\
Body mass index & -0.028 & 0.590 & 0.011 & 0.851 \\
Duration of diabetes & 0.193 & 0.0002 & 0.080 & 0.104 \\
Body weight gain $\left(\Delta \mathrm{BW}_{\text {max-20y }}\right)$ & -0.160 & 0.002 & -0.112 & 0.034 \\
Average systolic blood pressure & 0.166 & 0.001 & 0.213 & $<0.0001$ \\
Hemoglobin A1c & 0.065 & 0.209 & 0.013 & 0.800 \\
Total cholesterol & -0.121 & 0.019 & -0.084 & 0.094 \\
Logarithm of triglyceride & 0.049 & 0.346 & 0.034 & 0.508 \\
Uric acid & 0.139 & 0.010 & -0.040 & 0.456 \\
Creatinine & 0.042 & $<0.0001$ & 0.470 & $<0.0001$ \\
Smoking status & - & - & 0.101 & 0.062 \\
Dipeptidyl peptidase-4 inhibitor & - & - & 0.145 & 0.003 \\
RAS inhibitors & - & - & -0.024 & 0.632 \\
Statin & - & - & -0.053 & 0.283 \\
\hline
\end{tabular}

Sex was defined as female $(=0)$ or male $(=1)$, smoking status was defined as nonsmoker $(=0)$, past smoker $(=1)$, or current smoker $(=2)$, and medication for renin-angiotensin system (RAS) inhibitors or dyslipidemia was defined as without $(=0)$ or with $(=1)$.

On the other hand, we did not evaluate lean-muscle mass in our study. Little change in BW might be the result of less lean-muscle mass, which is associated with less insulin sensitivity and consequently an unfavorable metabolism. It is well known that the Asian population has less insulin sensitivity than the Western population [20]. Unmeasured insulin sensitivity might be an important residual confounder among patients with little change in BW from early adulthood.

Nonalcoholic fatty liver disease (NAFLD) is a known risk factor for albuminuria [21]. We previously reported the impact of NAFLD on incident type 2 diabetes in nonoverweight individuals [22]. Nonoverweight individuals with NAFLD had a higher risk of incident type 2 diabetes than overweight individuals without NAFLD. Moreover, the nonoverweight individuals with NAFLD who developed type 2 diabetes showed little change in BW from baseline to the time of incident type 2 diabetes. The presence of NAFLD in patients with little change in BW from early adulthood might have affected our findings.

There were several limitations to this study. First, this study had a cross-sectional design with retrospective components. More specifically, it took into account the difference between the reported BW at the age of 20 years and maximum BW that was reached through lifetime at some point until this study was conducted. The correlation analysis between UAE (the biological effect of processes that develop over time) and risk factors took only 1-point measurements into account. Second, it has been reported that weight loss can reverse renal pathology. The relationship between weight changes and changes in UAE maybe explained by the effect of weight change on blood pressure and cholesterol. Unfortunately, however, we had no information about the age of the patient and the duration of the maximum weight. Third, sarcopenia, which is associated with a loss of lean-muscle mass, could result in a lower BW; unfortunately, there was no data about sarcopenia. Fourth, the study population consisted only of Japanese men and women. Populations of Asian origin are reported to have lower BMI than those of European origin [23]; therefore, it is not certain whether our findings can be generalized to other ethnic groups. Fifth, medication adherence or medication for diabetes can affect UAE; we unfortunately had insufficient data on medication adherence and type of diabetes control medication. Finally, the number 


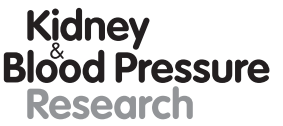

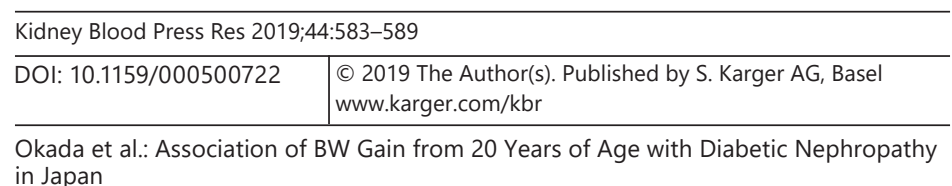

of patients available for analysis was not large enough. The BW information in this study was self-reported. Large prospective trials are needed to better assess the relationship between BW gain from early adulthood and albuminuria in patients with type 2 diabetes.

In conclusion, BW gain from the age of 20 years may be associated with diabetic nephropathy in Japanese patients with type 2 diabetes. Taking the potential effects of BW gain from the age of 20 years into consideration could be helpful in daily clinical practice.

\section{Statement of Ethics}

This study was approved by the local Research Ethics Committee. This study was performed according to the ethics statement by the Japanese Ministry of Health, Labor and Welfare, and was conducted the study in accordance with the Declaration of Helsinki. We provided information about the study, including aims, methods and handling of personal information, to patients based on the ethics statement by the Japanese Ministry of Health, Labor and Welfare, and patients provided verbal informed consent to participate. Data cannot be shared publicly. Data are available from the Ethics Committee (contact via Matsushita Memorial Hospital) for researchers who meet the criteria for access to confidential data.

\section{Disclosure Statement}

Michiaki Fukui reports grants from Japan Society for the Promotion of Science, AstraZeneca plc, Astellas Pharma Inc., Nippon Boehringer Ingelheim Co. Ltd., Daiichi Sankyo Co., Ltd., Eli Lilly Japan K.K., Kyowa Hakko Kirin Co. Ltd., Kissei Pharmaceutical Co. Ltd., MSD K.K., Mitsubishi Tanabe Pharma Corp., Novo Nordisk Pharma Ltd., Sanwa Kagaku Kenkyusho Co. Ltd., Sanofi K.K., Ono Pharmaceutical Co. Ltd., and Takeda Pharmaceutical Co. Ltd. outside the submitted work. The sponsors were not involved in the study design; collection, analysis, interpretation of data; writing of this manuscript; or the decision to submit the article for publication. The authors, their immediate families, and any research foundations with which they are affiliated have not received any financial payments or other benefits from any commercial entity related to the subject of this article.

\section{Funding Sources}

The authors declare that, although they are affiliated with a department that is supported financially by pharmaceutical companies, they received no current funding for this study and this does not alter their adherence to all the journal policies on sharing data and materials.

\section{Author Contributions}

All persons who meet authorship criteria are listed as authors, and all authors certify that they have participated sufficiently in the work to take public responsibility for the content.

H.O. researched data and wrote the manuscript. G.H., H.N., M.K., M.T., and Y.O. contributed to the discussion. K.N. and T.Y. researched data and contributed to the discussion. M.F. reviewed and edited the manuscript. H.O. is the guarantor of this work and, as such, had full access to all the data in the study and takes responsibility for the integrity of the data and the accuracy of the data analysis. 
Kidney
Blood Pressure
Research

\begin{tabular}{l|l}
\hline Kidney Blood Press Res 2019;44:583-589 \\
\hline DOI: 10.1159/000500722 & $\begin{array}{l}\text { ○ 2019 The Author(s). Published by S. Karger AG, Basel } \\
\text { www.karger.com/kbr }\end{array}$ \\
\hline
\end{tabular}

Okada et al.: Association of BW Gain from 20 Years of Age with Diabetic Nephropathy in Japan

\section{References}

1 Isomaa B, Almgren P, Tuomi T, Forsén B, Lahti K, Nissén M, et al. Cardiovascular morbidity and mortality associated with the metabolic syndrome. Diabetes Care. 2001 Apr;24(4):683-9.

2 Multiple Risk Factor Intervention Trial Research Group. Multiple risk factor intervention trial. Risk factor changes and mortality results. JAMA. 1982 Sep;248(12):1465-77.

3 Drury PL, Ting R, Zannino D, Ehnholm C, Flack J, Whiting M, et al. Estimated glomerular filtration rate and albuminuria are independent predictors of cardiovascular events and death in type 2 diabetes mellitus: the Fenofibrate Intervention and Event Lowering in Diabetes (FIELD) study. Diabetologia. 2011 Jan;54(1):32-43.

4 Dinneen SF, Gerstein HC. The association of microalbuminuria and mortality in non-insulin-dependent diabetes mellitus. A systematic overview of the literature. Arch Intern Med. 1997 Jul;157(13):1413-8.

5 Willett WC, Manson JE, Stampfer MJ, Colditz GA, Rosner B, Speizer FE, et al. Weight, weight change, and coronary heart disease in women. Risk within the 'normal' weight range. JAMA. 1995 Feb;273(6):461-5.

6 Manson JE, Colditz GA, Stampfer MJ, Willett WC, Rosner B, Monson RR, et al. A prospective study of obesity and risk of coronary heart disease in women. N Engl J Med. 1990 Mar;322(13):882-9.

7 Kurth T, Gaziano JM, Berger K, Kase CS, Rexrode KM, Cook NR, et al. Body mass index and the risk of stroke in men. Arch Intern Med. 2002 Dec;162(22):2557-62.

8 Chan JM, Rimm EB, Colditz GA, Stampfer MJ, Willett WC. Obesity, fat distribution, and weight gain as risk factors for clinical diabetes in men. Diabetes Care. 1994 Sep;17(9):961-9.

9 Colditz GA, Willett WC, Stampfer MJ, Manson JE, Hennekens CH, Arky RA, et al. Weight as a risk factor for clinical diabetes in women. Am J Epidemiol. 1990 Sep;132(3):501-13.

10 Stamler J. Epidemiologic findings on body mass and blood pressure in adults. Ann Epidemiol. 1991 May; 1(4): 347-62.

11 Chen Y, Copeland WK, Vedanthan R, Grant E, Lee JE, Gu D, et al. Association between body mass index and cardiovascular disease mortality in east Asians and south Asians: pooled analysis of prospective data from the Asia Cohort Consortium. BMJ. 2013 Oct;347:f5446.

12 Kong KA, Park J, Hong SH, Hong YS, Sung YA, Lee H. Associations between body mass index and mortality or cardiovascular events in a general Korean population. PLoS One. 2017 Sep;12(9):e0185024.

13 Murakami Y, Huxley RR, Lam TH, Tsukinoki R, Fang X, Kim HC, et al; Asia Pacific Cohort Studies Collaboration. Diabetes, body mass index and the excess risk of coronary heart disease, ischemic and hemorrhagic stroke in the Asia Pacific Cohort Studies Collaboration. Prev Med. 2012 Jan;54(1):38-41.

14 Carnethon MR, De Chavez PJ, Biggs ML, Lewis CE, Pankow JS, Bertoni AG, et al. Association of weight status with mortality in adults with incident diabetes. JAMA. 2012 Aug;308(6):581-90.

15 Bello AK, de Zeeuw D, El Nahas M, Brantsma AH, Bakker SJ, de Jong PE, et al. Impact of weight change on albuminuria in the general population. Nephrol Dial Transplant. 2007 Jun;22(6):1619-27.

16 Expert Committee on the Diagnosis and Classification of Diabetes Mellitus. Report of the expert committee on the diagnosis and classification of diabetes mellitus. Diabetes Care. 2003 Jan;26 Suppl 1:S5-20.

17 American Diabetes Association. Diagnosis and classification of diabetes mellitus. Diabetes Care. 2012 Jan;35 Suppl 1:S64-71.

18 American Diabetes Association. Standards of medical care in diabetes - 2012. Diabetes Care. 2012 Jan;35 Suppl 1:S11-63.

19 Naik RG, Brooks-Worrell BM, Palmer JP. Latent autoimmune diabetes in adults. J Clin Endocrinol Metab. 2009 Dec;94(12):4635-44.

20 Kodama K, Tojjar D, Yamada S, Toda K, Patel CJ, Butte AJ. Ethnic differences in the relationship between insulin sensitivity and insulin response: a systematic review and meta-analysis. Diabetes Care. 2013 Jun;36(6):178996.

21 Jia G, Di F, Wang Q, Shao J, Gao L, Wang L, et al. Non-Alcoholic Fatty Liver Disease Is a Risk Factor for the Development of Diabetic Nephropathy in Patients with Type 2 Diabetes Mellitus. PLoS One. 201513;10:e0142808.

22 Fukuda T, Hamaguchi M, Kojima T, Hashimoto Y, Ohbora A, Kato T, et al. The impact of non-alcoholic fatty liver disease on incident type 2 diabetes mellitus in non-overweight individuals. Liver Int. 2016 Feb;36(2):275-83.

23 Chan JC, Malik V, Jia W, Kadowaki T, Yajnik CS, Yoon KH, et al. Diabetes in Asia: epidemiology, risk factors, and pathophysiology. JAMA. 2009 May;301(20):2129-40. 